Ann. Biol. anim. Bioch. Biophys., 1977, 17 (5 B), 875-886.

\title{
Effets des lipides alimentaires sur les aspects qualitatifs de la lipogenèse chez le rat et le porc en croissance.
}

\author{
par Y. DEMARNE *, C. PERAZA-CASTRO, Y. HENRY **, J. FLANZY*. \\ * Station de Recherches de Nutrition \\ ** Station de Recherches sur l'Elevage des Porcs \\ I. N. R. A., 78350 jouy-en-josas.
}

Summary. Effects of dietary lipids on qualitative aspects of lipogenesis in growing rat and pig.

As compared to studies on very low-lipid diets ingested by growing rat and pig, the degree of deposit unsaturation may be increased by nutritional route; large amounts of different types of unsaturated fatty acids then appear in triglyceride stores. On the other hand, it is very difficult by this method to increase their degree of saturation and saturated long fatty acid content.

In the first part of this report we show the results of studies confirming this observation. The second part deals with the particular effects of some middle-chain fatty acids on the qualitative aspects of lipogenesis during growth.

In the young weaned rat, we compared long-term effects of the intake of two middlechain triglycerides, glycerol trioctanoate (Tri C $8: 0$ ) and glycerol tridodecanoate (Tri $\mathrm{C} 12$ : 0 ). As compared to the results of feeding a lipid-free diet, glycerol trioctanoate did not change the fatty acid composition of body lipid stores, but glycerol tridodecanoate was a nutritional factor deeply affecting this characteristic. As opposed to octanoic acid, high amounts of dodecanoic acid were incorporated into iriglyceride stores. They had a depressive action on the appearance of octadecenoic cis-9 acid (C18 $=1 \omega 9)$ and thus increased the ratio saturated fatty acids/unsaturated fatty acids in lipid deposits.

The intake of glycerol tridodecanoate gave similar results in growing pig. However, dodecanoic acid seemed to present a higher capacity for elongation into tetradecanoic acid $(\mathrm{C} 12: 0 \rightarrow \mathrm{C} 14: 0)$ in rat.

The metabolic effects of dodecanoic acid intake were already apparent before weaning. The young weaned rats from mothers fed glycerol tridodecanoate during growth, pregnancy and lactation, presented body lipids containing more than 25 p. 100 dodecanoic acid. This observation was associated to the fact that in these nutritional conditions, that fatty acid was incorporated at high amounts in the lipids secreted by the mammary gland.

The metabolic effects of glycerol tridodecanoate intake therefore should be totally different than those of other middle-chain triglycerides having a lower molecular weight, as glycerol trioctanoate, for example.

\section{Introduction.}

Dans l'adipocyte du mammifère monogastrique en croissance, et en dehors du cas particulier des acides gras qui sont régulièrement recyclés au sein même de cette 
cellule, les acides gras qui arrivent sous forme d'acyl-coenzyme A aux sites de biosynthèse des triglycérides de réserve peuvent être répartis entre trois grands « pools» :

- Le premier «pool» concerne les acides gras provenant d'une biosynthèse totale au niveau de la cellule adipeuse ou/et de la cellule hépatique.

- Le second « pool » concerne les acides gras ayant une origine exogène direcie, c'est-à-dire ceux qui proviennent des triglycérides alimentaires.

- Enfin, le dernier « pool» regroupe les acides gras ayant une origine exogène, ef ayant subi des transformations biochimiques, en particulier au niveau de l'adipocyte et/ou de l'hépatocyte (il s'agit surtout d'élongations et de désaturations).

Au cours de la croissance, et lorsque l'alimentation est contrôlée, l'équilibre qui s'éfablit entre ces trois pools conduit à l'obtention de triglycérides de réserve caractéristiques des conditions nutritionnelles dans lesquelles ils ont été élaborés. La façon dont s'établit cet équilibre, si elle dépend des disponibilités énergétiques globales au niveau cellulaire, dépend pour beaucoup de la qualité de cette énergie, et plus particulièrement de la composition en acides gras des lipides exogènes. Il en résulfe qu'à un même niveau d'apport d'énergie digestible les caractéristiques des tissus adipeux produits, et entre autres les compositions en acides gras des triglycérides de réserve, peuvent être extrêmement différentes (Demarne et al., 1974 ; Flanzy, Français et Rérat, 1970).

Dans ces conditions, on entrevoit la possibilité d'agir par voie nutritionnelle sur les aspects qualitatifs de la lipogenèse, et d'orienter dans différentes espèces animales la biosynthèse des triglycérides de réserve vers une certaine composition en acides gras, de façon à répondre à des critères qualitatifs de production précis, d'ordre technologique, organoleptique voire même thẻrapeutique. Cependant, il paraît nécessaire de déterminer les limites de cette méthode de contrôle qualitatif, tant dans le sens de l'augmentation du degré d'insaturation des dépôts que dans celui de sa diminution, et en se plaçant dans des conditions d'élevage compatibles avec des vitesses de croissance élevées et des indices de consommation bas.

Dans le cadre de cette présentation, nous rapportons la synthèse de différents travaux que nous avons entrepris afin d'étudier, chez le Rat et chez le Porc, les contrôles qui peuvent être exercés par voie nutritionnelle sur les aspecis qualitatifs de la lipogenèse pendant la croissance et sur leurs limites. De plus, nous attirons tout particulièrement l'attention sur les effets nutritionnels de certains acides gras à chaîne moyenne.

\section{Résultats.}

Effets des régimes lipidoprives ou contenant peu de lipides.

Les résultats qui sont enregistrés pendant la croissance en utilisant des régimes qui contiennent peu de lipides (couverture des besoins en acides gras essentiels) permettent d'obtenir une image représentative de la production d'acides gras résultant de la biosynthèse totale, réalisée essentiellement à partir des produits de la glycolyse, et que l'on peut considérer comme étant uniquement contrôlée par des informations d'ordre génétique. 
Dans ces conditions, comme on peut le voir sur le tableau 1, les triglycérides de réserve synthétisés par le Rat et par le Porc se caractérisent par leur richesse en acide hexadécanoïque ou palmitique (C16: 0 ; de 25 à 30 p. 100 des acides gras totaux) ef surtout en acide cis-9 octadécénoïque ou oléique (C18:1; de 45 à 55 p. 100 des acides gras totaux).

\section{TABLEAU 1}

Composition en ocides gros des lipides de réserve chez le raf ou le porc ayant reçu des régimes à basse teneur en matière grasse $(\leqslant 2$ p. 100). (Valeurs extrêmes rencontrées dans la littérature exprimées en p. 100 des acides gras totaux)

\begin{tabular}{lrr}
\hline Acides gras & \multicolumn{1}{c}{ Rat } & \multicolumn{1}{c}{ Porc } \\
\hline C14:0 & 1,0 ò 3,0 & 0,5 à 2,0 \\
$C 16: 0$ & 25,0 à 30,0 & 25,0 à 32,0 \\
C16:1 & 10,0 à 15,0 & 1,5 d̀ 5,0 \\
$C 18: 0$ & 3,0 à 4,0 & 10,0 à 20,0 \\
C18:1 & 40,0 d̀ 50,0 & 40,0 d̀ 55,0 \\
C18:2 & 3,0 à 10,0 & 2,0 à 9,0 \\
\hline
\end{tabular}

Effets des régimes à haute teneur en acides gras mono ef polyinsaturés.

D'une façon générale, ef bien que l'on ne domine pas totalement les différentes interactions entre acides gras d'origine exogène et endogène, on sait que les compositions des triglycérides de réserve synthétisés par l'animal pendant la croissance sont influencées par celle des triglycérides qui ont été introduits dans la ration alimentaire. Il y a donc tout intérêt à introduire dans l'alimentation des acides gras correspondant au type de triglycérides que l'on désire produire (François et Flanzy, 1959).

Ce moyen de contrôle se révèle très efficace quand il s'agit d'augmenter le degré d'insaturation des triglycérides de réserve.

Sur le tableau 2 nous rapportons les résultats d'analyse de lipides corporels de Rat et de Porc ayant ingéré pendant toute leur croissance différentes matières grasses se caractérisant par leur richesse en acides gras mono et polyinsaturés.

Chez le Rat, les deux exemples présentés, par comparaison avec ce qui est observé en cas d'ingestion d'un régime à faible teneur en lipides, montrent qu'il est possible d'augmenter de plus de 10 points les teneurs en acides gras insaturés des dépôts. En cas d'ingestion d'une quantité importante d'acide oléique (C18:1 de l'huile d'olive), cet acide gras peut représenter plus de 60 p. 100 des acides gras totaux ; l'effet compensateur se fait sentir à la fois sur l'acide palmitique et sur l'acide palmitoléique (C16:1 $1 \omega 7)$.

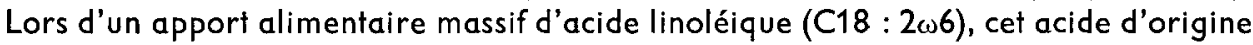
exclusivement exogène peut représenter jusqu'à 45 p. 100 des acides gras totaux. Son apparition à des concentrations très élevées dans les triglycérides de réserve est associée avec une diminution des teneurs en acide palmitique (acide gras saturé), mais aussi en acide oléique (acide gras insaturé), ce qui permet d'équilibrer en partie les concentrations en acides gras insaturés dans la carcasse. 
TABLEAU 2

Essais d'augmentation du degré d'insaturation des lipides des dépôts par introduction d'acides gras longs ef insaturés dans l'alimentation du raf ef du porc. (Valeurs exprimées en p. 100 des acides gras totaux)

\begin{tabular}{|c|c|c|c|c|c|c|}
\hline Acides gras & $\begin{array}{c}\text { Rat } \\
\text { (huile } \\
\text { d'olive) }\end{array}$ & $\begin{array}{l}\text { Rat } \\
\text { (huile de } \\
\text { tournesol) }\end{array}$ & $\begin{array}{c}\text { Rat } \\
\text { (témoin) }\end{array}$ & $\begin{array}{l}\text { Porc* } \\
\text { (huile } \\
\text { de soja) }\end{array}$ & $\begin{array}{l}\text { Porc* } \\
\text { (témoin) }\end{array}$ & $\begin{array}{l}\text { Porc* } \\
\text { (huile } \\
\text { de colza) }\end{array}$ \\
\hline $\begin{array}{l}C 14: 0 \ldots \ldots \ldots \ldots \\
C^{C} 16: 0 \quad \ldots \ldots \ldots \ldots\end{array}$ & $\begin{array}{r}1,6 \\
20,6\end{array}$ & $\begin{array}{r}1,2 \\
17,2\end{array}$ & $\begin{array}{r}2,7 \\
26,9\end{array}$ & $\begin{array}{r}1,4 \\
18,6\end{array}$ & $\begin{array}{r}1,7 \\
28,8\end{array}$ & $\begin{array}{r}1,7 \\
16,5\end{array}$ \\
\hline$C 16: 1\left\{\begin{array}{l}+ \\
\omega 9\end{array}\right.$ & 4,8 & 3,5 & 14,1 & 0,9 & 2,8 & 1,8 \\
\hline $\mathrm{C} 18: 0 \underset{j \omega 7}{\ldots} \cdots \cdots \cdots$ & 3,7 & 4,7 & 3,2 & 11,5 & 17,3 & 7,5 \\
\hline C18: $1\left\{\begin{array}{l}+ \\
\omega 9\end{array}\right.$ & 62,2 & 28,4 & 41,2 & 27,6 & 43,1 & 32,3 \\
\hline $\begin{array}{l}\text { C18 : } 2 \omega 6 \ldots \ldots \ldots \ldots \\
\text { Autres } \ldots \ldots \ldots \ldots \ldots \ldots\end{array}$ & $\begin{array}{l}5,8 \\
1,3\end{array}$ & $\begin{array}{r}43,7 \\
1,3\end{array}$ & $\begin{array}{l}9,9 \\
2,0\end{array}$ & $\begin{array}{r}35,4 \\
4,6\end{array}$ & $\begin{array}{l}6,1 \\
0,2\end{array}$ & $\begin{array}{l}18,1 \\
22,1 * *\end{array}$ \\
\hline Saturés $\ldots \ldots \ldots \ldots$. & 25,9 & 23,1 & 33,8 & 32,2 & 47,8 & 26,1 \\
\hline Insaturés $\ldots \ldots \ldots \ldots$. & 74,1 & 76,9 & 66,2 & 67,8 & 52,2 & 73,9 \\
\hline
\end{tabular}

* gras dorsal.

** dont $C 18: 3 \omega 3=6,0 \quad C 20: 1 \omega 9=8,3 \quad C 22: 1 \omega 9=7,8$.

Chez le Porc, au niveau du gras dorsal, les effets observés avec l'huile de soja sont comparables à ce qui est observé chez le Rat avec l'huile de tournesol. Cependant,

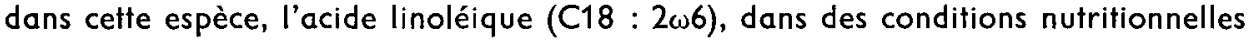
similaires, représente dans le tissu adipeux des concentrations plus faibles que chez le Rat, mais l'effet compensateur apparaît au niveau de tous les autres acides gras insaturés ou saturés des dépôts et avec la même amplitude (environ - 35 p. 100). En cas d'ingestion d'une quantité similaire d'un acide gras à chaîne longue monoinsaturé en $\omega 9$, l'acide érucique $(\mathrm{C} 22: 1 \omega 9)$ de l'huile de colza, cel acide gras totalement exogène n'est pas introduit directement dans les dépôts adipeux à des concentrations aussi élevées que l'acide linoléique. Cependant, les triglycérides obtenus présentent une composition en acides gras tout à fait caractéristique, hautement insaturée, et contenant des chaînes longues (> C18).

Dans les limites ci-dessus mentionnées, il apparaît done relativement aisé de modifier très nettement les teneurs ef les compositions en acides gras insaturés des lipides des dépôts adipeux du jeune mammifère en introduisant des acides gras de ce type dans son alimentation. Il est même possible de choisir le type d'acide gras insaturé que l'on désire voir apparaître en forte concentration dans le tissu adipeux (monoinsaturés en $\omega 9$ ou polyinsaturés en $\omega 6$ par exemple).

Effets des régimes à haute teneur en acides gras longs et saturés.

A la différence de ce qui est observé avec les régimes riches en acides gras insaturés, l'emploi de régimes riches en acides gras saturés et longs apparaît comme très 
peu efficace quand on désire augmenter le degré de saturation des triglycérides de réserve.

Par rapport à ce qui est observé en cas d'ingestion d'un régime à basse teneur en lipides (tabl. 3), l'introduction dans la ration d'une matière grasse très saturée comme le beurre de cacao ne conduit pas chez le Rat en croissance à une modification sensible des teneurs totales en acides gras saturés et longs (C16:0 et C18:0). II a fallu à Bollinger et Reiser (1965) assurer pendant plus d'un mois un apport quotidien massif de tripalmitate de glycérol (plus de 30 p. 100 de l'apport énergétique total) pour augmenter d'environ 10 points les concentrations en acides gras longs et safurés des lipides de réserve du Rat en croissance.

TABLEAU 3

Essais d'augmentation du degré de saturation des lipides des dépôts par introduction d'acides gras longs et safurés dans l'alimentation du rat en croissance (Valeurs exprimées en p. 100 des acides gras totaux)

\begin{tabular}{|c|c|c|c|c|c|}
\hline Acides gras & $\begin{array}{l}\text { Aliment } \\
\text { Beurre } \\
\text { de cacao * }\end{array}$ & $\begin{array}{c}\text { Rat } \\
\text { Beurre } \\
\text { de cacao }\end{array}$ & $\begin{array}{c}\text { Rat } \\
\text { Témoin }\end{array}$ & $\stackrel{\text { Rat }}{\text { Tri C16:0** }}$ & $\begin{array}{c}\text { Rat } \\
\text { Témoin ** }\end{array}$ \\
\hline $\begin{array}{l}C 14: 0 \ldots \ldots \ldots \ldots \\
C 16: 0 \quad \ldots \ldots \ldots \ldots \\
\text { C16 : } 1 \omega 7\end{array}$ & $\overline{32,5}$ & $\begin{array}{r}1,4 \\
22,9\end{array}$ & $\begin{array}{r}2,6 \\
26,9\end{array}$ & $\begin{array}{r}1,6 \\
37,9\end{array}$ & $\begin{array}{r}1,6 \\
28,0\end{array}$ \\
\hline $\mathrm{C} 16: 1 \omega 9$ & - & 4,0 & 14,1 & 10,5 & 11,4 \\
\hline $\begin{array}{l}\text { C18 }: 0 \ldots \ldots \ldots \ldots \\
\text { C18 }: 1 \omega 7 \\
\quad+\end{array}$ & 38,1 & 11,6 & 3,2 & 3,8 & 3,1 \\
\hline $\begin{array}{l}\text { C18 : } 1 \omega 9 \\
\text { C18 : } 2 \omega 6 \ldots \ldots \ldots \ldots \\
\text { Autres } \ldots \ldots \ldots \ldots\end{array}$ & $\begin{array}{r}26,1 \\
3,3 \\
-\end{array}$ & $\begin{array}{r}52,3 \\
5,3 \\
2,5\end{array}$ & $\begin{array}{r}44,2 \\
6,9 \\
2,0\end{array}$ & $\begin{array}{r}41,3 \\
3,0 \\
1,9\end{array}$ & $\begin{array}{r}50,5 \\
4,6 \\
0,8\end{array}$ \\
\hline Saturés & 70,6 & 35,9 & 32,8 & 43,3 & 32,7 \\
\hline Insaturés & 29,4 & 64,1 & 67,2 & 56,7 & 67,3 \\
\hline
\end{tabular}

* Beurre de cacao $=10$ p. 100 pondéral

** D'après Bollinger et Reiser (1965) - Trihexanoate de glycérol = 15 p. 100 pondéral.

Cette différence entre acides gras longs saturés et acides gras longs et insaturés doit être associée dans un premier temps aux coefficients d'utilisation digestive bas des acides gras saturés, ce qui constitue une première défense de l'organisme contre une invasion massive par des molécules de ce type (Demarne ef al., 1970 ; Flanzy, Rérat et François, 1968).

Elle doit également être associée au fait que l'acide palmitique, en particulier, représente normalement le stade ultime de la biosynthèse « de novo» des acides gras par le système « acide gras synthétase » du cytoplasme (Wakil, 1961). En effet, par le jeu des mécanismes de régulation de la biosynthèse des acides gras, l'aice palmitique exogène inhibe la synthèse d'acide palmitique «endogène» (Bortz et Lynen,1963). 
Au niveau des microsomes et des mitochondries, les acides gras saturés exogènes ( $C 16: 0$ et $C 18: 0$ ) qui n'ont pas été catabolisés par $\beta$-oxydation peuvent se substituer aux acides gras saturés endogènes et subir sous forme d'acyl-coenzyme A élongation et/ou désaturation conduisant à l'acide oléique (Harlan et Waki!, 1963). Ceci expliquerait que sur le plan qualitatif peu de différences apparaîtraient en cas d'ingestion d'acides gras de ce type ou en cas d'ingestion d'un régime sans lipides.

Effets des régimes contenant des acides gras à chaîne moyenne.

Quand l'animal en croissance ne reçoit pas de lipides dans sa ration, et que sa lipogenèse dépend totalement du système de biosynthèse « de novo» du cytoplasme, les acides gras à chaînes plus courtes que 16 atomes de carbone ne sont pas libérés par le système « acide gras synthétase » et n'apparaissent qu'en très faibles proportions dans les triglycérides de réserve (Bollinger et Reiser, 1965 ; Flanzy, François et Rérat, 1970). Pourtant ces acides gras sont d'excellents substrats énergétiques que l'organisme animal sait parfaitement ef efficacement métaboliser (Bach, Metais et Warter, 1968 ; Metais, Bach et Warter, 1967).

Au cours d'expériences de longue durée, on peut introduire des quantités importantes de triesters homogènes d'acides gras à chaîne moyenne et de glycérol, et déterminer ainsi l'influence de différents acides gras de ce type sur l'élaboration des réserves adipeuses au cours de la croissance. Dans ces conditions nutritionnelles, les effets obtenus varient non seulement en fonction de la longueur de chaîne, mais également en fonction de l'espèce animale étudiée.

Le tableau 4 rapporte une comparaison des effets obtenus à long terme avec un acide gras saturé en $\mathrm{C} 8$ : l'acide octanoïque ou caprylique $(\mathrm{C} 8: 0)$ et avec un acide

TABLEAU 4

Comparaison des effets de l'ingestion de Tri C12, de Tri C8 ef de régime lipidoprive sur la composition en ocides gras des lipides de la carcasse du rat en croissance *

\begin{tabular}{|c|c|c|c|}
\hline Acides gras & $\begin{array}{c}\text { Tri C12 } \\
\text { (10 p. 100/MS) }\end{array}$ & $\begin{array}{c}\text { Tri C8 } \\
\text { (10 p. 100/MS) }\end{array}$ & Lipidoprive \\
\hline 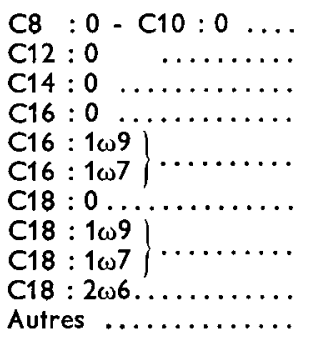 & $\begin{array}{r}\text { tr. } \\
28,1 \\
6,1 \\
22,7 \\
10,4 \\
2,8 \\
21,4 \\
6,0 \\
2,5\end{array}$ & $\begin{array}{r}1,3 \\
0,5 \\
2,8 \\
24,1 \\
14,1 \\
3,6 \\
40,3 \\
9,7 \\
3,6\end{array}$ & $\begin{array}{r}\text { tr. } \\
0,6 \\
2,7 \\
26,9 \\
14,4 \\
3,2 \\
41,2 \\
9,9 \\
1,1\end{array}$ \\
\hline $\begin{array}{l}\text { Acides gras salurés } \\
\Sigma C 8: 0 \text { à } C 12: 0 \ldots \\
\Sigma C 14: 0 \text { à } C 20: 0 \ldots\end{array}$ & $\left.\begin{array}{l}28,1 \\
31,7\end{array}\right\} 59,8$ & $\left.\begin{array}{r}1,8 \\
30,5\end{array}\right\} 32,3$ & $\left.\begin{array}{r}0,6 \\
32,8\end{array}\right\} 33,4$ \\
\hline
\end{tabular}

* Animaux abattus à $300 \mathrm{~g}$ et ayant reçu les différents régimes depuis leur sevrage. 
gras saturé en C12 : l'acide dodécanoïque ou laurique (C12 : 0) lorsqu'ils sont introduits dans l'alimentation du Rat en croissance.

En cas d'ingestion de tricaprylate de glycérol, les lipides de réserve présentent une composition en acides gras similaire à celle qui est observée en cas d'ingestion d'un régime sans lipide. L'acide caprylique n'apparaît pas en quantité importante dans les dépôts adipeux. Ceci confirme un certain nombre d'observations antérieures (Aurousseau et De Groot, 1972 ; Bollinger, 1963 ; Powell, 1930) et montre que cet acide gras est incapable d'entraîner, même à très long terme, une quelconque modification des aspects qualitatifs de la lipogenèse « de novo».

En revanche, l'ingestion d'acide laurique modifie profondément la composition en acides gras des dépôts adipeux du Rat en croissance. Cet acide gras s'y retrouve à des concentrations voisines de 30 p. 100 des acides gras totaux, et par rapport à ce qui est observé en cas d'ingestion du régime à basse feneur en lipide, on note également l'apparition d'une quantité importante d'acide tétradécanoïque ou myristique (C14:0) provenant très certainement de l'élongation de l'acide laurique. Cependant, le fait le plus intéressant est que l'ingestion prolongée d'acide laurique conduit à une diminution de l'ordre de 50 p. 100 des concentrations en acide oléique (C18:1). Dans ces conditions nutritionnelles les concentrations en acides gras saturés totaux à point de fusion supérieure à $40^{\circ} \mathrm{C}$ sont environ deux fois plus élevées qu'en cas d'ingestion d'un régime à basse teneur en lipide. Ils représentent ici 60 p. 100 des acides gras totaux des réserves lipidiques corporelles.

\section{TABLEAU 5}

Effets de l'introduction de trilaurate de glycérol dans la ration du porc en croissance, de 29 d̀ $90 \mathrm{~kg}$. Analyses effectuées sur les animaux abattus à $90 \mathrm{~kg}$ de poids vif (p. 100 des esters méthyliques dosés dans les lipides totaux des différents tissus adipeux étudiés)

\begin{tabular}{|c|c|c|c|c|c|c|}
\hline \multirow{2}{*}{ Acides gras } & \multicolumn{2}{|c|}{$\begin{array}{l}\text { Lard dorsal } \\
\text { externe }\end{array}$} & \multicolumn{2}{|c|}{ Périrénal } & \multicolumn{2}{|c|}{ Poitrine } \\
\hline & Tri C12* & $\mathrm{Lp}^{* *}$ & Tri C12* & $L_{p} * *$ & Tri C12* & $L_{p} * *$ \\
\hline \multirow{3}{*}{ 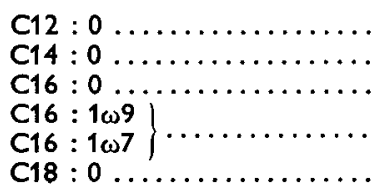 } & $\begin{array}{r}8,2 \\
12,8 \\
29,7\end{array}$ & $\begin{array}{r}0,2 \\
1,7 \\
26,4\end{array}$ & $\begin{array}{r}8,3 \\
14,4 \\
33,1\end{array}$ & $\begin{array}{r}0,2 \\
1,9 \\
30,5\end{array}$ & $\begin{array}{r}7,5 \\
13,7 \\
29,2\end{array}$ & $\begin{array}{r}0,2 \\
2,0 \\
28,0\end{array}$ \\
\hline & 8,1 & 4,0 & 4,6 & 1,8 & 7,8 & 4,0 \\
\hline & 8,0 & 13,6 & 13,9 & 22,2 & 9,6 & 14,1 \\
\hline \multirow{2}{*}{$\begin{array}{l}C 18: 1 \omega 9 \\
C 18: 1 \omega 7 \\
C 18: 2 \omega 6\end{array}$} & 27,9 & 45,2 & 20,9 & 38,5 & 26,8 & 46,2 \\
\hline & 5,3 & 8,9 & 4,8 & 4,9 & 5,4 & 5,5 \\
\hline $\begin{array}{l}\Sigma C 12: 0+C 14: 0 \ldots \ldots \\
\Sigma C 16: 0+C 18: 0 \ldots \ldots \\
\Sigma \text { Acides gras saturés } \ldots \ldots\end{array}$ & $\begin{array}{l}21,0 \\
37,7 \\
58,7\end{array}$ & $\begin{array}{r}1,9 \\
40,0 \\
41,9\end{array}$ & $\begin{array}{l}22,7 \\
47,0 \\
69,7\end{array}$ & $\begin{array}{r}2,1 \\
52,7 \\
54,8\end{array}$ & $\begin{array}{l}21,2 \\
38,8 \\
60,0\end{array}$ & $\begin{array}{r}2,2 \\
42,1 \\
44,3\end{array}$ \\
\hline$\sum M$. I. en $\omega 7$ et $\omega 9 . \ldots \ldots \ldots$ & 36,0 & 49,2 & 25,5 & 40,3 & 34,6 & 50,2 \\
\hline
\end{tabular}

* et ** : Rations à base de céréales et de tourteau de soja contenant $\left({ }^{*}\right)$ ou ne contenant pas (**) de Tri C12 (15 p. 100 par rapport à la matière sèshe). 
Quand on introduit du trilaurate de glycérol dans l'alimentation du Porc en croissance, et que l'on compare la composition en acides gras des lipides de réserve de différents tissus adipeux à ce qui est observé en cas d'ingestion d'un régime sans lipides, on observe également de profondes modifications. Sur le tableau 5 , on peut tout d'abord remarquer que, dans ce cas, l'acide laurique se retrouve dans les tissus adipeux, mais à des concentrations toujours inférieures à 10 p. 100 des acides gras totaux. L'apparition d'acide laurique dans les triglycérides de réserve est associée avec celle d'acide myristique (C14:0), à des concentrations variant entre 10 et 15 p. 100

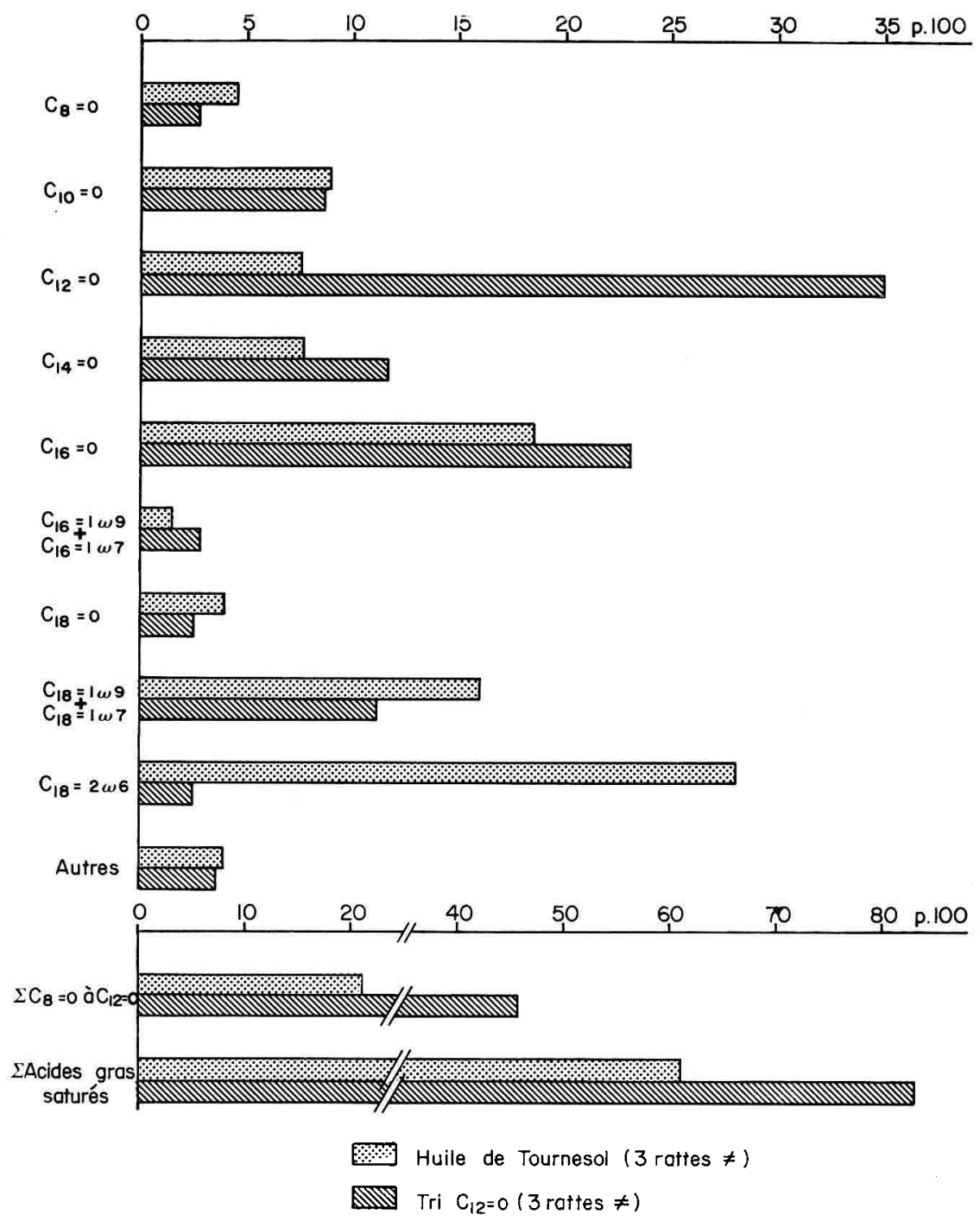

FIG. 1. - Effets de l'ingestion de trilourate de glycérol ou d'huile de tournesol sur la composition en ocides gras des lipides totaux du lait chez la rafte. 
des acides gras totaux. Les teneurs en acide palmitique (C16:0) ne sont pas modifiées par l'apport exogène d'acide laurique. En revanche, les teneurs en acide stéarique (C18:0) sont plus basses qu'en cas d'ingestion d'un régime sans lipides.

L'ingestion prolongée d'acide laurique par le Porc en croissance conduit à une diminution d'environ 50 p. 100 de la concentration en acide oléique (C18:1) dans les triglycérides de réserve et, d'une façon plus générale, conduit à une diminution importante du degré d'instauration des lipides de réserve.

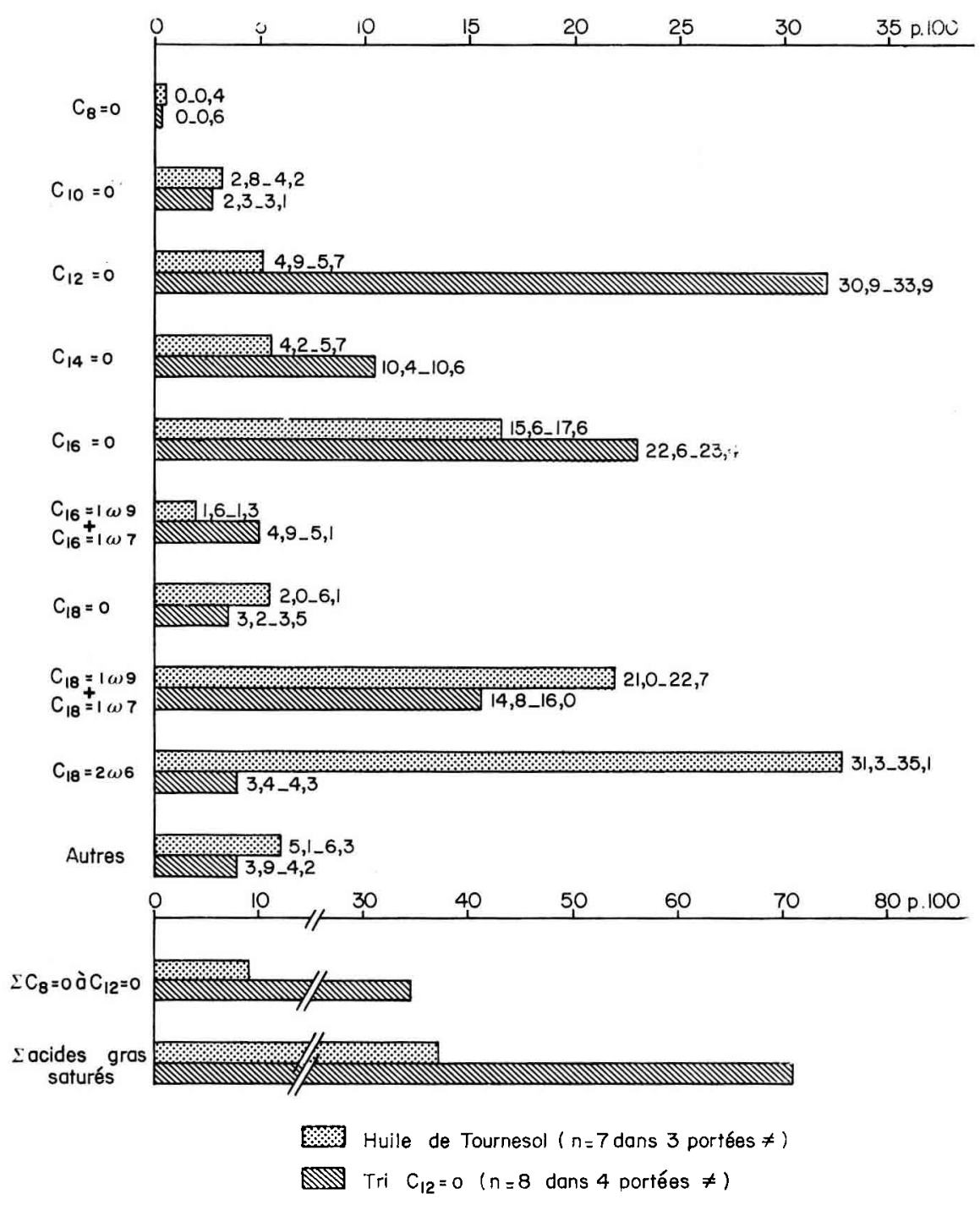

FIG. 2. - Effets de l'ingestion par la mère de trilaurate de glycérol ou d'huile de tournesol sur la composition en acides gras des lipides totaux corporels du jeune raton au sevrage. 
Quand on compare les effets dus à l'ingestion d'acide laurique au cours de la croissance chez le Rat et chez le Porc, les résultats obtenus se différencient selon l'espèce étudiée.

Ceci semble se traduire en particulier per une estérification directe de l'acide laurique dans les triglycérides plus élevées chez le Rat, eł par l'apparition d'une quanfité d'acide myristique plus importante chcz le Porc, reflétant sans doute une activité d'élongation plus élevée dans cette espèce. En revanche, l'effet dépressif de l'acide laurique exogène sur la mise en réserve d'acide oléique endogène paraît indépendant de l'espèce animale étudiée.

Effets de l'alimentation maternelle sur la lipogenèse du jeune avant le sevrage.

Peu de travaux ont été consacrés à l'étude de ce sujet. Nous rapportons sur les figures 1 et 2 les résultats d'une étude comparative des effets respectifs de l'huile de tournesol et du trilaurate de glycérol sur la sécrétion d'acides gras par la mamelle chez la Ratte et sur la composition en acides gras des lipides corporels du Raton au sevrage.

Ces résultats démontrent que les lipides alimentaires de la mère influencent profondément les aspects qualitatifs de la lipogenèse du jeune avant le sevrage. Nous examinerons plus particulièrement le cas de l'acide laurique.

Sur la figure 1, on peut observer qu'en cas d'ingestion de trilaurate de glycérol, l'acide laurique n'est pas uniquement introduit dans les lipides de réserve. Chez les femelles allaitantes, il apparaît à des concentrations très élevées dans les lipides du lait puisqu'il peut représenter entre 30 et 35 p. 100 des acides gras totaux. Ceci conduit à la sécrétion par la mamelle de lipides très riches en acides gras saturés (environ 80 p. 100 des acides gras totaux.).

Comme conséquence de l'ingestion d'acide laurique par la mère, on peut voir sur la figure 2 que les dépôts adipeux du jeune raton sevré présentent des teneurs très élevées en cet acide gras et que les concentrations en acides gras saturés totaux sont d'environ 70 p. 100 , ce qui est deux fois plus élevé qu'en cas d'ingestion par la mère d'huile de tournesol.

\section{Conclusion.}

Les travaux dont nous avons discuté les résultats au cours de cette présentation illustrent de quelle façon, et dans quelles limites, il est possible d'exercer un contrôle par voie nutritionnelle sur les aspects qualitatifs de la lipogenèse chez le mammifère monogastrique en croissance. Ce contrôle, exercé par le choix des lipides introduits dans la ration, peut déjà être très efficace avant même le sevrage.

Nous retiendrons de l'ensemble de ces résultats que s'il est relativement facile d'accroître par voie nutritionnelle le degré d'insaturation des dépôts adipeux du jeune en croissance et de modifier les concentrations en tel ou tel acide gras mono ou polyinsaturé dans les triglycérides de réserve, il apparaît beaucoup plus difficile d'exercer un contrôle efficace sur la mise en dépôt des acides gras saturés longs. Il est en particulier très difficile d'augmenter les concentrations en acide palmitique dans les dépôts. 
Il nous paraît intéressant d'associer cette observation au fait que cet acide gras représente le produit terminal de la biosynthèse endogène par la voie de « novo » autour de laquelle s'articulent les principaux mécanismes de régulation quantitative et qualitative du métabolisme des lipides de réserve. Le maintien d'une concentration de l'ordre de 30 p. 100 dans les triglycérides synthétisés pourrait être un facteur important de cette régulation.

Sur le plan pratique, pour augmenter au cours de la croissance les teneurs en acides gras saturés à point de fusion supérieur à $40^{\circ} \mathrm{C}$, seul l'emploi de l'acide laurique peut permettre de mettre efficacement en échec les défenses métaboliques de l'animal dans ce domaine, et de produire ainsi des triglycérides dont les compositions en acides gras apparaissent comme tout à fait originales.

Enfin, et sur le plan fondamental, il nous paraît important de souligner à ce propos :

1) qu'il existe des différences interspécifiques intéressantes entre Rat et Porc pour ce qui concerne la biosynthèse des triglycérides en cas d'apport massif d'acide laurique au cours de la croissance.

2) que l'acide laurique introduit dans l'alimentation maternelle est susceptible de modifier sensiblement la qualité des lipides synthétisés par le jeune mammifère avant le sevrage.

Réunion Groupe Développement INRA/Productions animales Thiverval-Grignon, 14-15 avril 1976.

Remerciements, - Une partie des travaux dont nous avons présenté les résultats a bénéficié d'une aide financière dans le cadre de l'action thématique programmée INSERM no 19 « Lipogenèse chez le Mammifère ».

\section{Références}

AUROUSSEAU B., De GROOT L., 1972. Influence de l'acide caprylique sur le métabolisme des autres acides gras chez le Rat en croissance. Ann. Biol. anim. Bioch. Biophys., 12, 617-630.

BACH A., METAIS P., WARTER J., 1968. Comparaison par l'étude du 14-CO ${ }_{2}$ de l'air expiré de l'utilisation des graisses à acides gras longs, moyens et courts. Influence du support. C. $R$. Soc. Biol, 162, 247-251.

BOLLINGER J. N., 1963. The metabolism of fatty acids derived from dietary triglycerides. Ph. D. Thesis Texas, 74 PP.

BOLLINGER J. N., REISER R., 1965. The metabolic fate or fatty acids derived from dietary triglycerides. J. Am. Oil Chem. Soc., 42, 1130-1133.

BORTZ W. M., LYNEN F., 1963. The inhibition of acetyl-CoA carboxylase by long-chain acyl-CoA derivatives. Biochem. Z., 337, 505-509.

DEMARNE Y., SACQUET E., FLANZY J., GARNIER H., FRANÇOIS A. C., 1970. Utilisation digestive apparente des acides gras chez le rat axénique et le rat holoxénique. Ann. Biol. anim. Bioch. Biophys., 10, 369-384.

DEMARNE Y., TOURÉ M., FLANZY J., LECOURTIER M. J., 1974. Evolution des réserves lipidiques du jeune rat en croissance en fonction du temps et de la qualité des lipides ingérés. Ann. Biol. anim. Bioch. Biophys., 14, 793-811.

FLANZY J., FRANÇOIS A. C., RÉRAT A., 1970. Utilisation métabolique des acides gras chez le porc. Ann. Biol. anim. Bioch. Biophys., 10, 603-620. 
FLANZY J., RÉRAT A., FRANÇOIS A. C., 1968. Etude de l'utilisation digestive des acides gras chez le porc. Ann. Biol. anim. Bioch. Biophys., 8, 537-548.

FRANÇOIS A. C., FLANZY J., 1959. Données nouvelles sur l'influence de l'alimentation sur les graisses de réserve. Aspect qualitatif. Ann. Nut. Alim., 13, A111-A162.

HARLAN W. R., WAKIL S. J., 1963. Synthesis of fatty acids in animal tissues. Incorporation of C14Acetyl-CoA into a variety of long chain fatty acids by subcellular particles. J. biol. Chem., 238, 3216-3223.

METAIS P., BACH A., WARTER J., 1967. Comparaison, par l'étude du 14- $\mathrm{CO}_{2}$ de l'air expiré, de l'utilisation de graisses à acides gras longs, moyens ou courts, C. R. Soc. Biol. 161, 1372.

POWELL M., 1930. The metabolism of tricaprylin and trilaurin. J. biol. Chem., 89, 547-552.

WAKIL S. J., 1961. Mechanisms of fatty acid synthesis. J. Lipid Res., 2, 1-24. 